\title{
Reconstruction of Total Solar Irradiance by Deep Learning
}

\author{
Yasser Abduallah, ${ }^{1}$ Jason T. L. Wang, ${ }^{1}$ Yucong Shen, ${ }^{1}$ Khalid A. Alobaid, ${ }^{1}$ \\ Serena Criscuoli, ${ }^{2}$ Haimin Wang ${ }^{1}$ \\ ${ }^{1}$ New Jersey Institute of Technology, University Heights, Newark, New Jersey, USA \\ \{ya54,wangj,ys496,kaa65,haimin.wang\}@ njit.edu \\ ${ }^{2}$ National Solar Observatory, Boulder, Colorado, USA
}

\begin{abstract}
The Earth's primary source of energy is the radiant energy generated by the Sun, which is referred to as solar irradiance, or total solar irradiance (TSI) when all of the radiation is measured. A minor change in the solar irradiance can have a significant impact on the Earth's climate and atmosphere. As a result, studying and measuring solar irradiance is crucial in understanding climate changes and solar variability. Several methods have been developed to reconstruct total solar irradiance for long and short periods of time; however, they are physics-based and rely on the availability of data, which does not go beyond 9,000 years. In this paper we propose a new method, called TSInet, to reconstruct total solar irradiance by deep learning for short and long periods of time that span beyond the physical models' data availability. On the data that are available, our method agrees well with the state-of-the-art physics-based reconstruction models. To our knowledge, this is the first time that deep learning has been used to reconstruct total solar irradiance for more than 9,000 years.
\end{abstract}

\section{Introduction}

Deep learning has drawn significant interest in recent years. It has been used extensively in biomedical applications for Parkinson's disease assessment (Hammerla et al. 2015), drug-disease interaction learning (Chen and Li 2020), drugdrug interaction prediction (Lin et al. 2020), clinical event prediction (Qiao et al. 2018), breast cancer subtype classification (Rhee, Seo, and Kim 2018), medical image segmentation (Zhang et al. 2020b) and so on. It finds many applications in other domains as well. For example, deep learning has been used for power load forecasting (Chen et al. 2018) and traffic forecasting (Yu, Yin, and Zhu 2018). More recently, Zhang et al. (2020a) employed a periodic long shortterm memory (LSTM) network for parking behavior prediction. Jiang et al. (2020) used a U-shaped convolutional neural network (CNN) to track solar magnetic flux elements. In this paper, we propose a new deep learning method for reconstructing total solar irradiance.

Solar irradiance is the primary source of energy for our Earth (Kren, Pilewskie, and Coddington 2017), and is a key input for climate models and changes (Ball et al. 2011). It is described in terms of total solar irradiance (TSI) when

Copyright (C) 2021 1 , Association for the Advancement of Artificial Intelligence (www.aaai.org). All rights reserved. all of the radiation is measured. Irradiance is defined as the amount of light energy from an object that is hitting a square meter of another object each second. Solar irradiance is the amount of light energy from the Sun's entire disk measured at the Earth, and it is known to vary over different temporal scales, in a manner that is strongly wavelength dependent (Kopp 2016). TSI variability affects the Earth's atmosphere and climate in many ways (Gray et al. 2010). To understand the effect of solar radiation on our Earth's climate changes, solar irradiance records for long periods of time are required. Since systematic measurements of irradiance started only in the late seventies, many models were introduced to provide irradiance records dating back to times ranging from century to millennia. All such models are based on the empirical evidence that irradiance variability is modulated by surface magnetism (Domingo et al. 2009), while the approaches adopted in the different models are mostly driven by the type of proxies of the magnetic field available at the temporal scales considered.

Most of the published models aim to reconstruct irradiance variability up to a few centuries into the past (Lean 2000; Krivova, Balmaceda, and Solanki 2007). Such models are intended to address the impact of solar variability on Earth's increase of temperatures registered from the preindustrial era, and mostly make use of sunspot, or sunspotgroup number, as a proxy of the surface magnetic activity. A few models have instead been proposed in the literature aiming at reconstructing irradiance variations at longer temporal scales. Because at those times telescopic observations were not available, such reconstructions necessarily make use of indirect proxies. These mostly consist of radioisotopes like ${ }^{14} \mathrm{C},{ }^{10} \mathrm{Be}$ and nitrate-related species (Usoskin 2017), which are generated by the interaction of energetic particles with the Earth's atmosphere, whose flux, in turn, is regulated by the heliospheric magnetic field.

Some of the historical irradiance reconstruction models used linear regression relationships between the irradiance measured at modern times and input proxies. More complex techniques make use of geomagnetic models to estimate from radioisotopes the open and closed components of the solar magnetic field, from which the distribution of magnetic features over the Sun's disk is recovered. The most recent state-of-the-art model of this kind was developed by $\mathrm{Wu}$ et al. (2018a), who reconstructed TSI for the previous 9 millen- 
nia, making use of two different cosmogenic isotopes, ${ }^{10} \mathrm{Be}$ and ${ }^{14} \mathrm{C}$, derived from various datasets (Wu et al. 2018b). All of the published models reconstructed solar irradiance based on physics properties (Wu et al. 2018a).

In this paper, we present the first deep learning model, called TSInet, to reconstruct total solar irradiance for more than 9,000 years. Our main contributions are outlined as follows:

1. We use TSInet to reconstruct TSI for the entire 9,000 years already covered in the recent reconstruction by $\mathrm{Wu}$ et al. (2018a) and for additional 1,000 years when physical data are not available.

2. Our deep learning model does not rely on proxies; hence our model is not affected by uncertainties in the proxies including errors in their measurements and estimates.

3. Our TSInet method can be extended back at times when proxies are not available. When physical data are available, TSInet agrees well with the state-of-the-art physicsbased reconstruction models on the available data.

\section{Data}

In this work, we use measurements of the TSI provided by the Total Irradiance Monitor aboard the SOlar Radiation and Climate Experiment (SORCE)(Rottman 2005) and available at http: / / lasp.colorado. edu / home/sorce/data/tsi-data/. This dataset, used as our training set, contains daily TSI measurements carried out from 2003 to the present. Figure 1 illustrates the SORCE time series dataset showing the total solar irradiance over time.

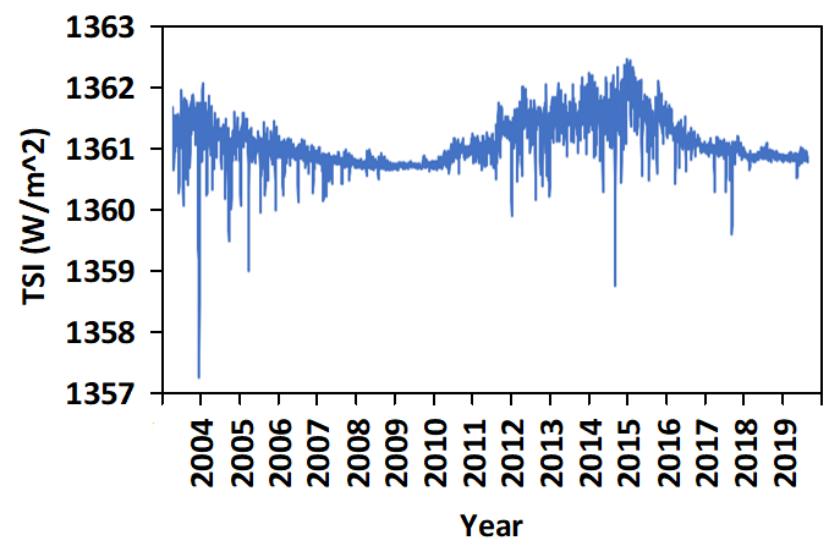

Figure 1: SORCE total solar irradiance (TSI) data from 2003 to the present.

Our testing set contains measurements from TCTE Total Solar Irradiance daily averages, available at http:// lasp.colorado.edu/lisird/data/tcte_tsi_ $24 \mathrm{hr} /$. Total Solar Irradiance Calibration Transfer Experiment (TCTE) (http: / / lasp. colorado.edu/home / missions-projects/quick-facts-tcte/) measurements are made by the LASP TCTE Total Irradiance Monitor (TIM) instrument aboard the U.S. Air Force's
STPSat-3 spacecraft. This TIM has been measuring total solar irradiance since late 2013.

In addition, we adopt the following publicly available datasets obtained, over different temporal ranges, by different physics-based models, which will be used as testing sets in our work.

- NRLTSI2 Daily Averages, available at http: / / lasp. colorado.edu/lisird/, is the daily climate record of total solar irradiance from 1882 to the present. It is constructed using version 2 of the Naval Research Laboratory's (NRL) solar variability model (NRLTSI2). The NRLTSI 2 model computes TSI based on the changes of the quiet Sun conditions arising from bright faculae and dark sunspots on the solar disk. It uses linear regression between solar sunspots and facular features, as well as irradiance observations from SORCE.

- SATIRE-S (Spectral And Total Irradiance REconstruction model - Space era), available at http: / / www2 .mps.mpg. de/projects / sun-climate/data.html, provides daily reconstruction of solar irradiance in the period of 1974 - 2013. Irradiance is reconstructed by combining the area coverage of magnetic and quiet features as derived by full-disk magnetograms and continuum images of the Sun, together with spectral syntheses obtained by one-dimensional, static, atmosphere models (Yeo et al. 2014).

- SATIRE-M (Spectral And Total Irradiance REconstruction model - Millennia), available at http: / / www2 .mps.mpg.de/projects / sun-climate/data.html, is similar to SATIRE-S, but the area coverage of magnetic structures is estimated by making use of a model which relies on the sunspot number (Wu et al. 2018b). This model provides decennial averages and reconstructs the solar irradiance over the last 9,000 years. The model is used to reconstruct decadal total TSI.

The total solar irradiance values range from 1356.656 to 1363.525. We use a feature scaling technique, also known as data normalization, to normalize the range of data to increase the cohesion of the TSI values. Specifically, we use the min-max normalization that is calculated as follows:

$$
\hat{v}_{i}=\frac{v_{i}-\min (S)}{\max (S)-\min (S)}
$$

where $\hat{v}_{i}\left(v_{i}\right.$, respectively) is the normalized value (actual value, respectively) at time point $i$, and $S$ represents the input data set. The normalized TSI values range from 0 to 1 .

\section{Proposed Method}

\section{Architecture and Training of TSInet}

Figure 2 presents the architecture of our TSInet. Let $t$ be the latest time point. Data sample $x_{t}$ contains $w$ values $v_{t}, v_{t-1}, \ldots, v_{t-w+1}$ and the label $v_{t-w}$ where $v_{t}$ is the TSI value at time point $t$. (In the study presented here, the time window, $w$, is set to 7.) We train TSInet with multiple batches. In the first batch, we use the $n$ training data 


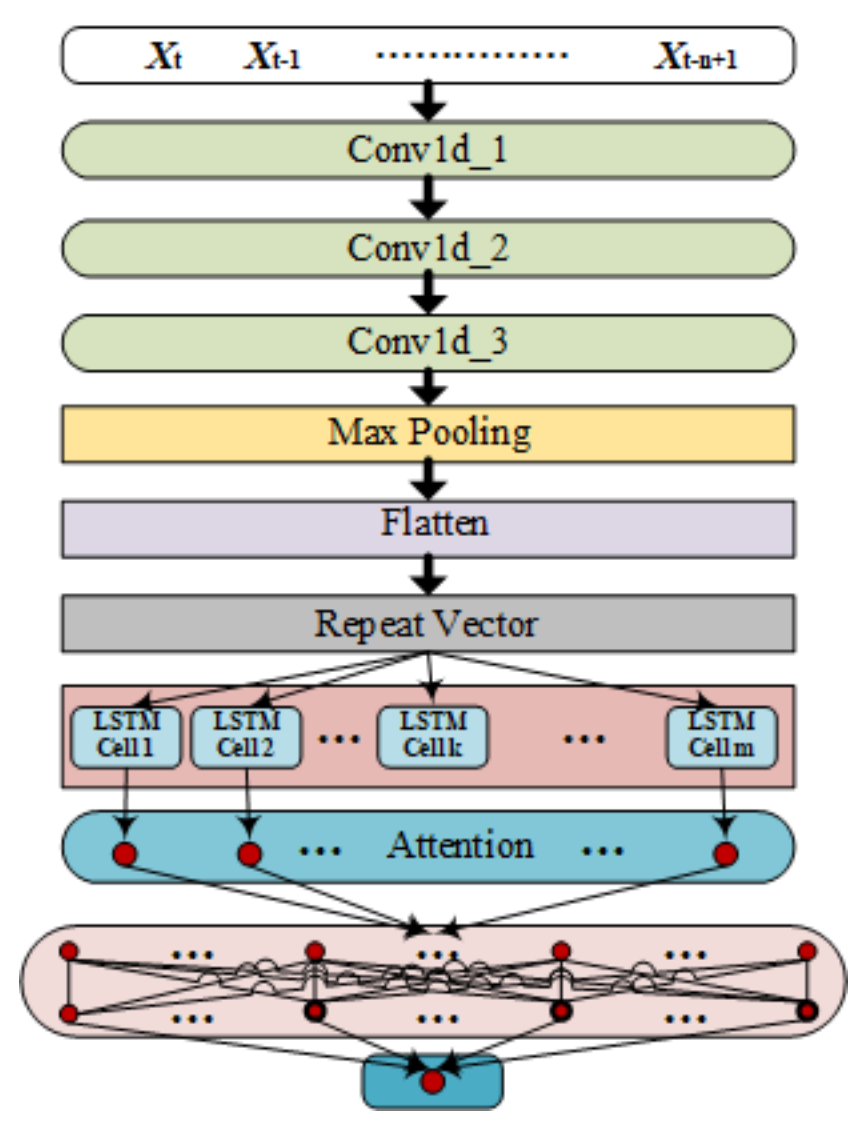

Figure 2: Architecture of TSInet.

samples, $x_{t}, x_{t-1}, \ldots, x_{t-n+1}$, to train TSInet. (In the study presented here, the number of input data samples, $n$, is set to 10.) The label of the $n$ training data samples is determined by the label of the last data sample (i.e., $x_{t-n+1}$ ). In the second batch, we use the next $n$ training data samples, $x_{t-n}, x_{t-n-1}, \ldots, x_{t-2 n+1}$, to train TSInet. The label of the $n$ training data samples is determined by the label of $x_{t-2 n+1}$. In the third batch, we use the following $n$ training data samples, $x_{t-2 n}, x_{t-2 n-1}, \ldots, x_{t-3 n+1}$, to train TSInet. The label of the $n$ training data samples is determined by the label of $x_{t-3 n+1}$. We continue this training process until all TSI values in the training set are used. For every two adjacent data samples $x_{i}, x_{i-1}$, they overlap on $w-1$ TSI values, namely $v_{i-1}, v_{i-2}, \ldots, v_{i-w+1}$.

TSInet consists of three convolutional layers (Conv1d_1, Conv1d_2, and Conv1d_3) where the kernel slides along 1 dimension on the time series, a max pooling layer, a flatten layer, a repeat vector layer, an LSTM (long short-term memory) layer, an attention layer, two fully connected layers, and an output layer. The output from the three convolutional layers is flattened by the flatten layer and transformed into a sequence, also known as a feature vector. The repeat vector layer repeats the feature vector to reshape and prepare it as the input to the LSTM layer. The LSTM layer in our architecture contains $m$ LSTM cells (in this study, $m$ is set to 10). The attention layer with $m$ neurons is used to focus on the relevant information in each time step (Bahdanau, Cho, and Bengio 2014). Each of the two fully connected layers has 200 neurons. The activation function used in our model is ReLU (rectified linear unit). TSInet produces as output a predicted TSI value.

The proposed TSInet is implemented in Python, Keras, and Tensorflow. We use adaptive moment estimation (Adam) (LeCun, Bengio, and Hinton 2015; Goodfellow, Bengio, and Courville 2016) as the network optimizer, which is a stochastic gradient descent algorithm that can update network weights based on training data. Adam is configured with a learning rate of 0.003 and a weight decay of 0.000005 to regularize the weights and minimize the test error during training in each epoch. Other Adam parameters $\left(\beta_{1}, \beta_{2}\right.$, respectively) are set to default values $(0.9,0.999$, respectively). To achieve faster back-propagation convergence, we adopt the mini-batch strategy described in (LeCun, Bengio, and Hinton 2015; Goodfellow, Bengio, and Courville 2016). The number of epochs is set to 10 by default.

\section{Reconstruction of Total Solar Irradiance}

After describing the architecture and training procedure of TSInet, we now turn to the algorithms for reconstructing total solar irradiance (TSI) in a testing set. We develop two reconstruction algorithms: (i) single-step or 1-step reconstruction; (ii) multi-step or $k$-step, $k>1$, reconstruction.

Let $t$ be the latest time point. With single-step reconstruction, we begin by considering the $n$ testing data samples $x_{t}, x_{t-1}, \ldots, x_{t-n+1}$ where $x_{t}$ contains the $w+1$ TSI values $v_{t}, v_{t-1}, \ldots, v_{t-w+1}, v_{t-w}$ in the testing set. Our TSInet model, which is trained as described in Section "Architecture and Training of TSInet," takes as input the $n$ testing data samples and predicts the label of the last testing data sample (i.e., $x_{t-n+1}$ ), which is treated as the label of the $n$ testing data samples. We then use the $n$ testing data samples together with the predicted label to re-fit or re-train TSInet. The re-trained TSInet then takes as input the next $n$ testing data samples $x_{t-1}, x_{t-2}, \ldots, x_{t-n}$ and predicts the label of the last testing data sample (i.e., $x_{t-n}$ ), which is treated as the label of the $n$ testing data samples. We again use the $n$ testing data samples together with the predicted label to re-fit or re-train TSInet. The re-trained TSInet then takes as input the following $n$ testing data samples $x_{t-2}, x_{t-3}, \ldots, x_{t-n-1}$ and predicts the label of the last testing data sample (i.e., $x_{t-n-1}$ ), which is treated as the label of the $n$ testing data samples. We then use the $n$ testing data samples together with the predicted label to re-train TSInet. We continue this predicting-retraining process until all TSI values (labels) in the testing set have been predicted, at which point we have reconstructed all the TSI values in the testing set.

With multi-step reconstruction, we begin by considering the first batch containing the $n$ testing data samples $x_{t}, x_{t-1}, \ldots, x_{t-n+1}$ in the testing set. Our trained TSInet takes as input these $n$ testing data samples and predicts the label of the last testing data sample (i.e., $x_{t-n+1}$ ), which is treated as the label of the $n$ testing data samples. Then, the same TSInet model takes as input the second batch contain- 
ing the next $n$ testing data samples $x_{t-1}, x_{t-2}, \ldots, x_{t-n}$ and predicts the label of the last testing data sample (i.e., $x_{t-n}$ ), which is treated as the label of the $n$ testing data samples. We keep on using the same TSInet model until the model takes as input the $k$ th batch containing the $n$ testing data samples $x_{t-k+1}, x_{t-k}, \ldots, x_{t-n-k+2}$ and predicts the label of the last testing data sample (i.e., $x_{t-n-k+2}$ ), which is treated as the label of the $n$ testing data samples. We then use the $k$ batches, where each batch contains $n$ testing data samples together with their predicted label, to retrain our TSInet as shown in Figure 2. Then we use the re-trained model to predict the labels for the next $k \times n$ testing data samples.

The difference between single-step reconstruction and multi-step reconstruction is that the former retrains TSInet once using one batch containing $n$ testing data sample in every one step while the latter retrains TSInet once using $k$ batches containing $k \times n$ testing data samples in every $k$ steps.

\section{Experiments and Results}

\section{Performance Metrics}

We conducted a series of experiments to evaluate the performance of the proposed TSInet and compare it with related methods. The performance metrics used here are the root mean square error (RMSE) and Pearson correlation coefficient (CORR). RMSE is calculated as follows:

$$
\operatorname{RMSE}=\sqrt{\frac{1}{n} \sum_{i=1}^{n}\left(\hat{y}_{i}-y_{i}\right)^{2}}
$$

where $\hat{y}_{i}\left(y_{i}\right.$, respectively) represents the predicted TSI value (actual TSI value, respectively) at time point $i$. RMSE measures the differences between the actual TSI values and the predicted TSI values by a method. The lower the RMSE value, the more accurate the method is. CORR is calculated as follows:

$$
\operatorname{CORR}=\frac{\sum_{i}\left(\hat{y}_{i}-\mu(\hat{y})\right)\left(y_{i}-\mu(y)\right)}{\sqrt{\sum_{i}\left(\hat{y}_{i}-\mu(\hat{y})\right)^{2}} \sqrt{\sum_{i}\left(y_{i}-\mu(y)\right)^{2}}}
$$

where $\mu(\hat{y})$ denotes the mean of all predicted TSI values and $\mu(y)$ denotes the mean of all actual TSI values. CORR is used to measure how strong the relationship between the predicted and actual TSI values is. CORR ranges from 1 to -1 , where 1 means there is a strong positive correlation, -1 means there is a strong negative correlation, and 0 means there is no correlation between the predicted and actual TSI values.

\section{Single-Step vs. Multi-Step Reconstruction Algorithms}

In this experiment, we compared the single-step (i.e., 1step) and multi-step (i.e., $k$-step) reconstruction algorithms described in Section "Reconstruction of Total Solar Irradiance." We used the SORCE training set to train TSInet and reconstructed the TSI values in the TCTE testing set for varying $k, k=1, \ldots, 10$. For each $k$, we computed the performance metrics and recorded the runtime used by the algorithms. Figure 3(i) shows the performance metrics, RMSE and CORR, for varying $k$. it can be seen from the figure that the performance of TSInet degrades as $k$ increases. This happens because the TSInet model is refitted more often, and hence is more accurate when $k$ is smaller. On the other hand, smaller $k$ requires more runtime, as shown in Figure 3(ii). In subsequent experiments, we fixed $k=5$ as it achieved good performance while requiring reasonable runtime.

\section{Ablation Tests}

In this experiment, we performed ablation tests to analyze and evaluate the components of our TSInet framework by considering two models based on TSInet: CNN and LSTM. The CNN model is a subnet of TSInet, keeping the three convolutional layers, max pooling layer, flatten layer, repeat vector layer, attention layer, two fully connected layers, and output layer, but removing the LSTM layer. The LSTM model is also a subnet of TSInet, keeping the LSTM layer, attention layer, two fully connected layers, and output layer, but removing the three convolutional layers, max pooling layer, flatten layer, and repeat vector layer.

Figure 3(iii) (Figure 3(iv), respectively) presents the RMSE (CORR, respectively) results from TSInet, CNN, and LSTM. It can be seen from the figures that TSInet yields the best accuracy and correlation among the three methods. This happens because CNN learns characteristics from the input data but it lacks temporal components to deeply analyze the time series information in the data. On the other hand, LSTM captures the temporal correlation in the input data, but it works on the raw input data without learning additional characteristics to strengthen the correlation between the data entries. TSInet combines the characteristics it learns in the CNN network and temporal correlation it learns in the LSTM network. Therefore, TSInet achieves the best performance.

\section{Comparison with Related Methods}

In this experiment, we compared TSInet with four closely related machine learning algorithms including linear regression (LR), Gaussian process regression (GPR) (Senanayake, O'Callaghan, and Ramos 2016; Al-Shedivat et al. 2017), random forest (RF), and support vector regression (SVR). Figures 3(v) and 3(vi) present the RMSE results for the five methods on the TCTE and NRLTSI 2 datasets respectively. The figures show that TSInet achieves the best performance among the five methods in terms of RMSE. The CORR results are similar and omitted here.

To assess whether the results obtained by our TSInet agree with entries in the testing datasets, we performed the Wilcoxon signed-rank test (Pratt 1959). According to the test, the difference between the TSInet results and entries in TCTE (NRLTSI2, SATIRE-S respectively) is not statistically significant with $p=0.01552<0.05(0.0001<0.05$, $0.0001<0.05$ respectively).

\section{Reconstruction of TSI on the SATIRE-M Dataset}

SATIRE-M contains decennial averages and is comprised of solar irradiance over the last 9,000 years. Each entry in the SATIRE-M dataset represents an average of 10 years. However, our TSInet reconstructs daily TSI. To reconstruct solar 


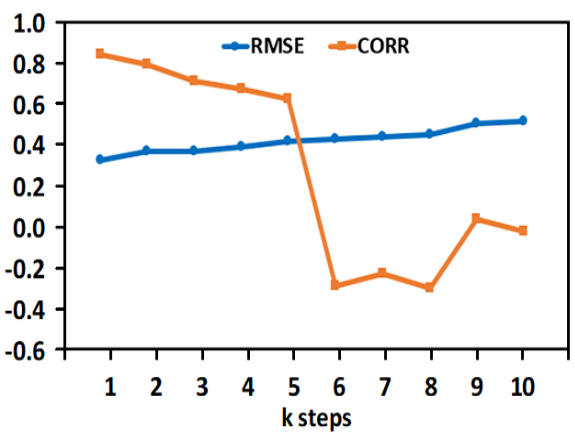

(i)

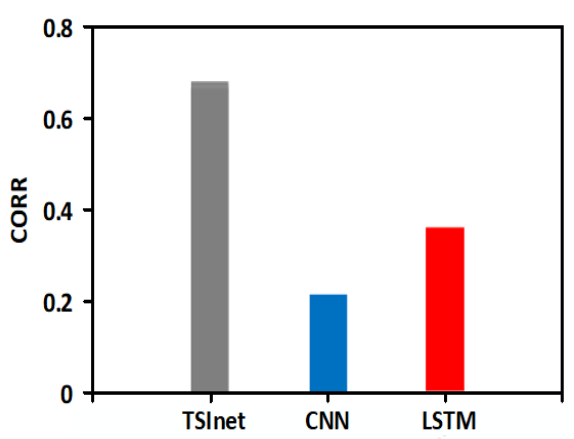

(iv)

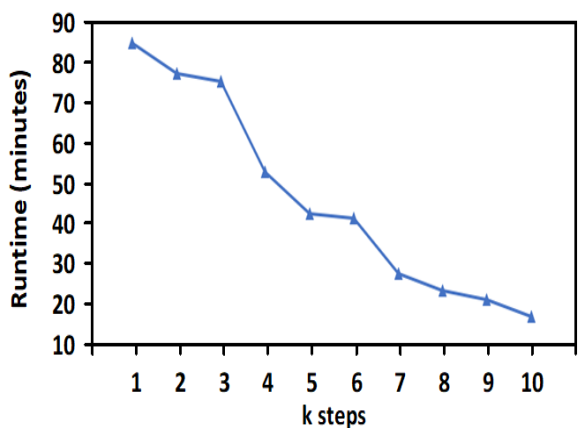

(ii)

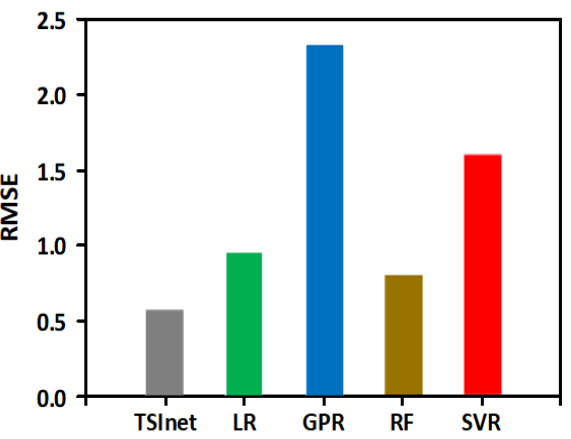

(v)

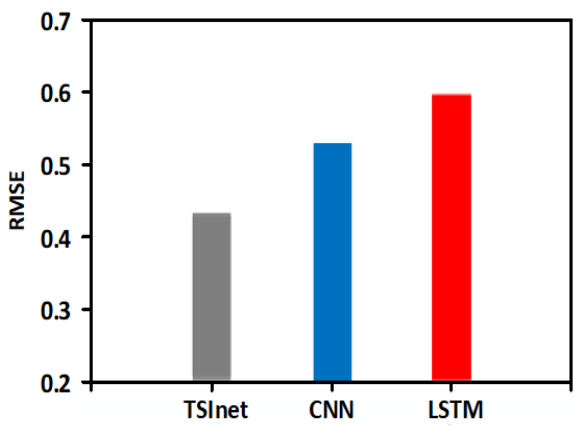

(iii)

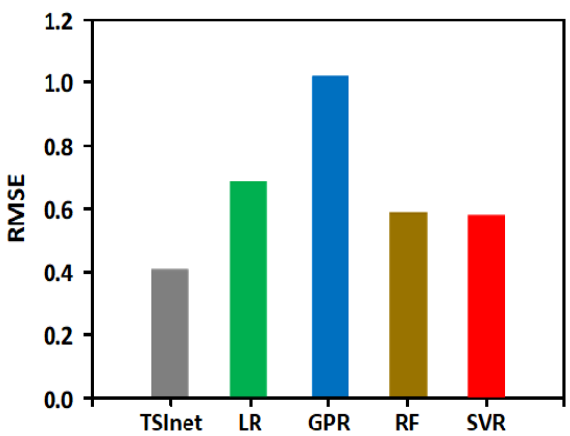

(vi)

Figure 3: (i) RMSE and CORR values obtained by TSInet for $k$-step, $k=1,2, \ldots, 10$, reconstruction of TSI on the TCTE dataset. (ii) Runtime needed by TSInet for $k$-step, $k=1,2, \ldots, 10$, reconstruction of TSI on the TCTE dataset. (iii) Comparison of RMSE values of TSInet, CNN and LSTM on the TCTE dataset. (iv) Comparison of CORR values of TSInet, CNN and LSTM on the TCTE dataset. (v) Comparison of RMSE values of five TSI reconstruction methods on the TCTE dataset. (vi) Comparison of RMSE values of five TSI reconstruction methods on the NRLTSI2 dataset. Results from the SATIRE-S dataset are similar and omitted.

irradiance on the SATIRE-M dataset, we employ the following technique. Recall that the SATIRE-S dataset provides daily reconstruction of solar irradiance in the period of 1974 - 2013. We first use TSInet to reconstruct total solar irradiance beyond 1974 on SATIRE-S. Then we take 10-year averages on the reconstructed TSI values.

Figure 4 compares the 10-year averages obtained by TSInet with the entries in SATIRE-M. TSInet's results agree mostly with entries in SATIRE-M. According to the Wilcoxon signed-rank test (Pratt 1959), the difference between TSInet's results and SATIRE-M entries is not statistically significant $(p=0.000297<0.05)$. Figure 4 also shows that our TSInet model is capable of reconstructing total solar irradiance beyond 9,000 years. We reconstructed total solar irradiance for additional 1,000 years beyond the SATIRE-M data.

\section{Conclusions}

The Earth's primary source of energy is the radiant energy from the Sun. This energy is known as solar irradiance, or total solar irradiance (TSI) when all of the radiation is measured. The changes in solar irradiance have a significant impact on Earths' atmosphere and climate. Therefore, study-

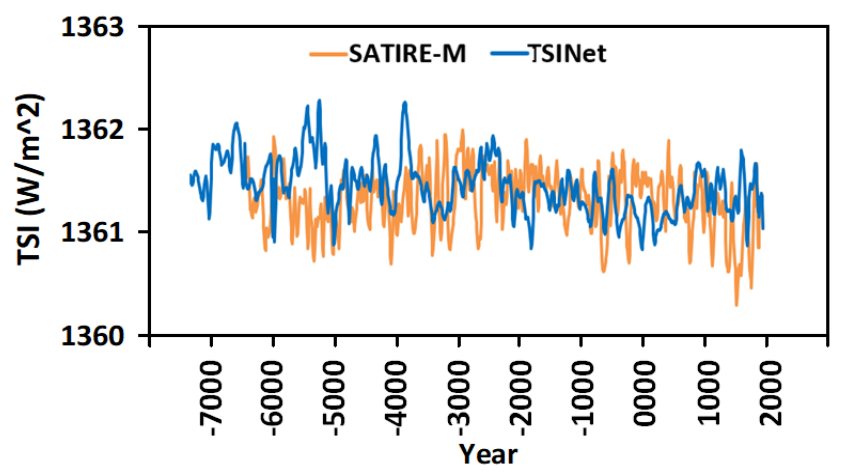

Figure 4: SATIRE-M reconstruction using TSInet plus reconstruction of solar irradiance for additional1,000 years.

ing and reconstructing solar irradiance is crucial in solar physics. Existing methods for solar irradiance reconstruction are all based on physics-based models (Wu et al. 2018a). In this paper, we presented the first deep learning method (TSInet) for reconstructing total solar irradiance (TSI). Experimental results showed that results from our TSInet agree 
well with those from the physics-based models. When compared to closely related machine learning methods, TSInet achieves the best performance among the methods. TSInet does not depend on physics properties such as proxies, and hence it can be extended back at times when proxies were not available. We demonstrated here that TSInet is able to reconstruct TSI for more than 9 millennia.

\section{References}

Al-Shedivat, M.; Wilson, A. G.; Saatchi, Y.; Hu, Z.; and Xing, E. P. 2017. Learning scalable deep kernels with recurrent structure. Journal of Machine Learning Research 18(1).

Bahdanau, D.; Cho, K.; and Bengio, Y. 2014. Neural machine translation by jointly learning to align and translate. CoRR abs/1409.0473.

Ball, W. T.; Unruh, Y. C.; Krivova, N. A.; Solanki, S.; and Harder, J. W. 2011. Solar irradiance variability: A six-year comparison between SORCE observations and the SATIRE model. Astronomy \& Astrophysics 530:A71.

Chen, H., and Li, J. 2020. Learning data-driven drug-targetdisease interaction via neural tensor network. In Proceedings of IJCAI 2020, 3452-3458.

Chen, P.; Liu, S.; Shi, C.; Hooi, B.; Wang, B.; and Cheng, X. 2018. Neucast: Seasonal neural forecast of power grid time series. In Proceedings of IJCAI 2018, 3315-3321.

Domingo, V.; Ermolli, I.; Fox, P.; Frohlich, C.; Haberreiter, M.; Krivova, N.; Kopp, G.; Schmutz, W.; Solanki, S. K.; Spruit, H. C.; Unruh, Y.; and Vogler, A. 2009. Solar surface magnetism and irradiance on time scales from days to the 11-year cycle. Space Science Reviews 145:337-380.

Goodfellow, I. J.; Bengio, Y.; and Courville, A. C. 2016. Deep Learning. MIT Press.

Gray, L. J.; Beer, J.; Geller, M.; Haigh, J. D.; Lockwood, M.; Matthes, K.; Cubasch, U.; Fleitmann, D.; Harrison, G.; Hood, L.; Luterbacher, J.; Meehl, G. A.; Shindell, D.; van Geel, B.; and White, W. 2010. Solar influences on climate. Reviews of Geophysics 48:RG4001.

Hammerla, N. Y.; Fisher, J.; Andras, P.; Rochester, L.; Walker, R.; and Ploetz, T. 2015. PD disease state assessment in naturalistic environments using deep learning. In Proceedings of AAAI, 1742-1748.

Jiang, H.; Wang, J.; Liu, C.; Jing, J.; Liu, H.; Wang, J. T. L.; and Wang, H. 2020. Identifying and tracking solar magnetic flux elements with deep learning. The Astrophysical Journal Supplement Series 250(1):5.

Kopp, G. 2016. Magnitudes and timescales of total solar irradiance variability. Journal of Space Weather and Space Climate 6:A30.

Kren, A. C.; Pilewskie, P.; and Coddington, O. 2017. Where does Earth's atmosphere get its energy? Journal of Space Weather and Space Climate 7:A10.

Krivova, N. A.; Balmaceda, L.; and Solanki, S. K. 2007. Reconstruction of solar total irradiance since 1700 from the surface magnetic flux. Astronomy \& Astrophysics 467(1):335-346.
Lean, J. 2000. Evolution of the Sun's spectral irradiance since the Maunder Minimum. Geophysical Research Letters 27(16):2425-2428.

LeCun, Y.; Bengio, Y.; and Hinton, G. 2015. Deep learning. Nature 521(7553):436-444.

Lin, X.; Quan, Z.; Wang, Z.; Ma, T.; and Zeng, X. 2020. KGNN: knowledge graph neural network for drug-drug interaction prediction. In Proceedings of IJCAI 2020, 27392745.

Pratt, J. W. 1959. Remarks on zeros and ties in the Wilcoxon signed rank procedures. Journal of the American Statistical Association 54(287):655-667.

Qiao, Z.; Zhao, S.; Xiao, C.; Li, X.; Qin, Y.; and Wang, F. 2018. Pairwise-ranking based collaborative recurrent neural networks for clinical event prediction. In Proceedings of IJCAI 2018, 3520-3526.

Rhee, S.; Seo, S.; and Kim, S. 2018. Hybrid approach of relation network and localized graph convolutional filtering for breast cancer subtype classification. In Proceedings of IJCAI 2018, 3527-3534.

Rottman, G. 2005. The SORCE mission. Solar Physics 230:7-25.

Senanayake, R.; O'Callaghan, S.; and Ramos, F. 2016. Predicting spatio-temporal propagation of seasonal influenza using variational Gaussian process regression. In Proceedings of AAAI 2016, 3901-3907.

Usoskin, I. G. 2017. A history of solar activity over millennia. Living Reviews in Solar Physics 14:3.

Wu, C. J.; Krivova, N. A.; Solanki, S. K.; and Usoskin, I. G. 2018a. Solar total and spectral irradiance reconstruction over the last 9000 years. Astronomy \& Astrophysics 620:A120.

Wu, C. J.; Usoskin, I. G.; Krivova, N.; Kovaltsov, G. A.; Baroni, M.; Bard, E.; and Solanki, S. K. 2018b. Solar activity over nine millennia: A consistent multi-proxy reconstruction. Astronomy \& Astrophysics 615:A93.

Yeo, K. L.; Krivova, N. A.; Solanki, S. K.; and Glassmeier, K. H. 2014. Reconstruction of total and spectral solar irradiance from 1974 to 2013 based on KPVT, SoHO/MDI, and SDO/HMI observations. Astronomy \& Astrophysics 570:A85.

Yu, B.; Yin, H.; and Zhu, Z. 2018. Spatio-temporal graph convolutional networks: A deep learning framework for traffic forecasting. In Proceedings of IJCAI 2018, 3634-3640.

Zhang, F.; Feng, N.; Liu, Y.; Yang, C.; Zhai, J.; Zhang, S.; He, B.; Lin, J.; and Du, X. 2020a. PewLSTM: Periodic LSTM with weather-aware gating mechanism for parking behavior prediction. In Proceedings of IJCAI 2020, 44244430.

Zhang, Q.; Liu, L.; Ma, K.; Zhuo, C.; and Zheng, Y. 2020b. Cross-denoising network against corrupted labels in medical image segmentation with domain shift. In Proceedings of IJCAI 2020, 1047-1053. 